\title{
Reprogramming rat embryonic fibroblasts into induced pluripotent stem cells using transposon vectors and their chondrogenic differentiation in vitro
}

\author{
JIAKUAN YE, JUNYI HONG and FUSHENG YE \\ Department of Orthopaedics, Xiaoshan Hospital, Hangzhou, Zhejiang 311202, P.R. China
}

Received December 1, 2013; Accepted July 9, 2014

DOI: $10.3892 / \mathrm{mmr} .2014 .2793$

\begin{abstract}
It has been known that the successful reprogramming of differentiated human somatic cells into a pluripotent state would allow for the creation of cartilage cells. However, current virus-mediated strategies to form induced pluripotent stem cells (iPSCs) are limited in their clinical application due to exogenous gene modification. In the present study, the piggyBac transposon system carrying corresponding genes (Oct3/4, Klf4, Sox 2 and c-Myc) was employed to reprogram rat embryonic fibroblasts (REFs) into iPSCs, and the transposon-carried genes were successfully removed by a transposase system. Furthermore, the differentiation capacity of the iPSCs into chondrocytes was investigated in vitro. A typical chondrocyte marker, collagen-II, was expressed following culture. In conclusion, rat iPSCs without genetic modification were obtained from REFs using the piggyBac transposon system and the transposase $\mathrm{mPB}$ system, and these cells possessed the capacity of chondrocyte differentiation, suggesting that this method may be an effective approach for the treatment of cartilage disorders in the future.
\end{abstract}

\section{Introduction}

Cartilage injuries remain a major clinical problem due to the poor self-healing ability of articular cartilage (1). Despite recent cell-engineering advances, the treatment of cartilage injuries has remained challenging. As chondrocytes have a limited capability of regeneration, it is necessary to obtain a sufficient number of chondrocytes in vitro for repairing traumatic joint surface lesions. Although stem cell transplantation therapy using mesenchymal stem cells (MSCs) has been considered a

Correspondence to: Dr Jiakuan Ye, Department of Orthopaedics, Xiaoshan Hospital, 728 Yu Cai Bei Road, Hangzhou, Zhejiang 311202, P.R. China

E-mail: jiakye@163.com

Key words: reprogramming, piggyBac transposon, induced pluripotent stem cells, mPB, chondrocyte prominent strategy, the major problem of limited proliferative capacity of autologous cells remains to be addressed (2).

It is well-established that the successful reprogramming of differentiated human somatic cells into a pluripotent state would allow for the creation of patient- and disease-specific stem cells. Since Takahashi et al (3) demonstrated that fibroblasts were reprogrammed into induced pluripotent stem cells (iPSCs) by defined transcription factors, numerous differentiation strategies have been applied to reprogram mature cells into a single- or pluripotent stem cells, including hematopoietic cells (4), cardiomyocytes (5), neural progenitors (6), liver cells (7) and hepatic stem cells (8). A number of studies have suggested iPSCs as alternative candidates for the treatment of numerous disorders (9-11). Previously, Teramura et al (2) demonstrated that the properties of iPSCs render them suitable alternative candidates for the treatment of articular disorders and suggested an effective approach for preparing chondrocytes from iPSCs.

Additionally, current strategies of virus-mediated formation of iPSCs are markedly limited in their clinical application $(12,13)$. Other approaches, including a plasmid transfection method, are able to obtain mouse iPSCs directly without exogenous gene modification (12). However, the efficiency of such strategies is markedly low and with poor operability, due to the low probability of its successful integration into the cellular genome (12). Previously, Yusa et al (13) described an efficient piggyBac transposon-based approach to generate integration-free iPSCs. The transposons carrying $2 \mathrm{~A}$ peptide-linked reprogramming factors induced reprogramming of mouse embryonic fibroblasts (MEF) with equivalent efficiencies to retroviral transduction. To date, there are a limited number of studies using this piggyBac transposon-based approach to reprogram rat embryonic fibroblasts (REF) to iPSCs without any genetic alteration (13).

Therefore, the aim of the present study was to apply a piggyBac transposon-based approach to reprogram REF into iPSCs, and subsequently to investigate its capacity of chondrocyte differentiation in vitro.

\section{Materials and methods}

Reprogramming of REF using transposon vectors. The present study was approved by the Ethics Committee of Fudan University (Shanghai, China). REF were isolated from embry- 
Table I. Primer sequences used for polymerase chain reaction.

\begin{tabular}{ll}
\hline Primer & \multicolumn{1}{c}{ Sequence } \\
\hline Oct4-F & 5'-CCCCATTTCACCACACTCTACTC-3' \\
Oct4-R & 5'-GTGACAGGAACAGAGGGAAAGG-3' \\
Nanog-F & 5'-GACTAGCAACGGCCTGACTCA-3' \\
Nanog-R & 5'-CTGCAATGGATGCTGGGATA-3' \\
Sox2-F & 5'-CTGTTTTTCATCCCAATTGCA-3' \\
Sox2-R & 5'-CGGAGATCTGGCGGAGAATA-3' \\
GAPDH-F & 5'-ATCACTGCCACTCAGAAG-3' \\
GAPDH-R & 5'-AAGTCACAGGAGACAACC-3' \\
IRx3-F & 5'-GCTCAATGAACACCGCAAGA-3' \\
IRx3-R & 5'-GTGATGATGGCCAACATGATCT-3' \\
Maf- F & 5'-CAGACGCTCCTCCTGAGCTT-3' \\
Maf- R & 5'-CATTCGTTGGGCACCAAAG-3' \\
Ramp2- F & 5'-CATCTGCCTCATCCCTTTCC-3' \\
Ramp2-R & 5'-TGCGCATCGCCGTCTT-3' \\
Sal3- F & 5'-CCAGCTGTCTCCGACCAGTT-3' \\
Sal3- R & 5'-TGACGTTTGCATAGAGTCAAGCA-3' \\
Hoxa5-F & 5'-CCCGGACTACCAGTTGCATAA-3' \\
Hoxa5-R & 5'-CGCCGAGTCCCTGAATTGT-3' \\
\hline
\end{tabular}

$\mathrm{F}$, forward; R, reverse.

onic day (E)14.5 Sprague-Dawley rat embryos. The pregnant rats were obtained from Shanghai Laboratory Animal Center, CAS (Shanghai, China). REFs were cultured in Dulbecco's modified Eagle's medium (DMEM; Hyclone, Logan, UT, USA) supplemented with $10 \%$ fetal bovine serum (FBS; Gibco-BRL, Carlsbad, CA, USA). For the generation of iPSCs, REF were plated onto a $35-\mathrm{mm}$ dish $\left(5 \times 10^{5}\right.$ cells per well) 1 day prior to transfection. The next day, $3 \mu \mathrm{g}$ pCMV-mPBase and plasmids pPB-CAG.OSKM-pu $\Delta$ tk or pPB-CAG.OSKML-pu $\Delta$ tk (donated by the Wellcome Trust Sanger Institute, Hinxton, UK) containing the piggyBac transposon were transfected using Lipofectamine 2000 (Invitrogen Life Technologies, Carlsbad CA, USA) according to the manufacturer's instructions. Following one day, the REF were trypsinized and re-plated onto L-wnt3a feeder layers at a ratio of 1:10 in REF medium. The medium was replaced every other day. Following seven days, the medium was changed to N2B27 medium [1:1 mixture of N2 medium (DMEM/F12, N2 supplement) and B27 medium (Neurobasal medium, B27 supplement)] supplemented with $0.1 \mathrm{mM}$ 2-mercaptoethanol (Invitrogen Life Technologies), $2 \mathrm{mM}$ L-glutamine (Invitrogen Life Technologies), $1 \mathrm{X}$ non-essential amino acids (EMD Millipore, Billerica, MA, USA) and 1,000 U/ml hLIF $1 \mathrm{mM}$ MEK1/2 (mitogen activated protein kinase kinase 1/2) inhibitor PD0325901 (Selleck Chemicals, Shanghai, China). Following 14 days, the colonies were stained using the alkaline phosphatase (AP) detection kit (Sigma-Aldrich, St. Louis, MO, USA) and counted, selected and further expanded.

Immunostaining. The cells were fixed in $4 \%$ paraformaldehyde in phosphate-buffered saline (PBS) for $20 \mathrm{~min}$ at room temperature and permeabilized in $0.1 \%$ Triton-X 100 in PBS
Table II. Primer sequences.

\begin{tabular}{ll}
\hline Gene & \multicolumn{1}{c}{ Sequence } \\
\hline L (Sox2-Klf4 junction)-F & $\begin{array}{l}\text { 5'-GCAACGGCAGCTACAG } \\
\text { CATGATGCAG-3' }\end{array}$ \\
L (Sox2-Klf4 junction)-R & $\begin{array}{l}\text { 5'-CAGGAGGTCGTTGAACT } \\
\text { CCTCGGTCTC-3' }\end{array}$ \\
R (PGK promoter)-F & 5'-GCGTCGACCGACTGTGC \\
& CTTCTAGTTGCCAGCC-3' \\
R (PGK promoter)-R & 5'-GTTGGCGCCTACCGGTG \\
& GATGTGGAATGTG-3' \\
Endogenous control-F & 5'-GGGGCCTTCTGGGGGTA \\
& AAGTTCAGAACAC-3' \\
Endogenous control-R & 5'-TGGCTGCCTGAGGGCA \\
& AGAGGGAAAGAATC-3' \\
\hline
\end{tabular}

F, forward; R, reverse.

Table III. Primer sequences.

\begin{tabular}{ll}
\hline Gene & \multicolumn{1}{c}{ Sequence } \\
\hline Aggrecan -F & 5'-GAAGTGGCGTCCAAACCAAC-3' \\
Aggrecan -R & 5'-AGCTGGTAATTGCAGGGGAC-3' \\
Sox9-F & 5'-TCCCCGCAACAGATCTCCTA-3' \\
Sox9-R & 5'-AGCTGTGTGTAGACGGGTTG-3' \\
Col2a1-F & 5'-GCTTCTGGTAACCCAGGGAC-3' \\
Col2a1-R & 5'-TTGGGGCCTTGTTCACCTTT-3' \\
GAPDH-F & 5'-ATCACTGCCACTCAGAAG-3' \\
GAPDH-R & 5'-AAGTCACAGGAGACAACC-3' \\
\hline
\end{tabular}

F, forward; R, reverse.

for $20 \mathrm{~min}$, then blocked with 5\% FBS in PBS for 40 min at room temperature. The cells were washed with PBS and incubated with the following primary antibodies: Goat polyclonal octamer-binding transcription factor 4 (Oct4; 1:100; sc-8628; Santa Cruz Biotechnology, Inc., Santa Cruz, CA, USA), rabbit polyclonal Nanog (1:300; ab80892; Abcam, Cambridge, UK), mouse monoclonal stage-specific embryonic antigen 1 (SSEA1; 1:200; sc-21702; Santa Cruz Biotechnology, Inc.) and His-tag mouse monoclonal immunoglobulin (Ig)G (1:200; ab18184; Abcam) overnight at $4^{\circ} \mathrm{C}$, followed by goat anti-mouse immunoglobulin IgM-fluorescein isothiocyanate (FITC) (1:400; Beyotime Institute of Biotechnology, Shanghai, China), donkey anti-goat IgG-FITC (1:40; Beyotime Institute of Biotechnology), goat anti-mouse IgG-FITC (1:400; Beyotime Institute of Biotechnology), goat anti-rabbit IgG-FITC or Cy3 (1:400; Beyotime Institute of Biotechnology) as secondary antibodies. Nuclei were stained with Hoechst 33258 (Sigma-Aldrich).

Quantitative polymerase chain reaction $(q P C R)$ and western blot analysis. Total RNA was extracted by using TRIzol reagent (Invitrogen Life Technologies). RNA was reverse-transcribed with random primer using SuperScript III (Takara Bio Inc., 

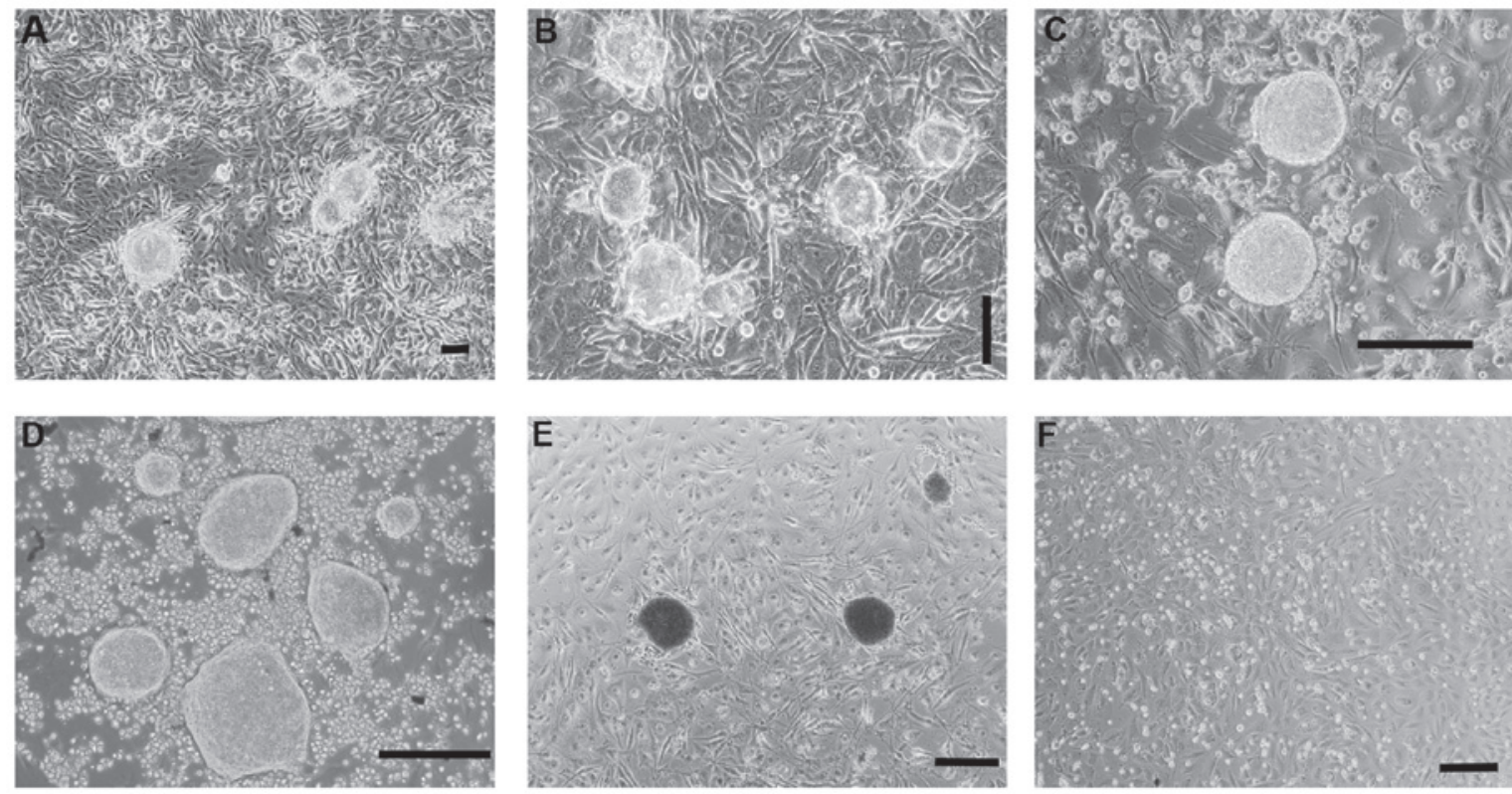

Figure 1. The formation of induced pluripotent stem cells on the L-wnt3a feed layer. (A) PB-OSKM-transfected REF on L-wnt3a feed layer following 14 days of culture (magnification, x100); (B) PB-OSKM-transfected REF supplemented with PD0325901 following 21 days of culture (magnification, x200); (C) PB-OSKM-transfected REF supplemented with PD0325901 at 35 days of culture (magnification, $\mathrm{x} 400$ ); (D) Rat ES as a positive control (magnification, $\mathrm{x} 400$ ); (E) alkaline phosphatase staining of the PB-OSKM transfected REF group (magnification, x100); (F) empty vehicle control group (magnification, x100). Scale bar, $100 \mu \mathrm{m}$. ES, embryonic stem cells; REF, rat embryonic fibroblasts.

Shiga, Japan) according to the manufacturer's instructions. qPCR was performed using SYBRgreen PCR Master Mix (Takara Bio Inc.) on the ABI7500 system (Applied Biosystems Life Technologies, Foster City, CA, USA) according to the manufacturer's instructions; the primers are listed in Table I. The expanded colonies were collected and suspended in radioimmunoprecipitation buffer (Sangon Biotech Co., Ltd., Shanghai, China) and the proteins were separated on electrophoresis gels. The protein blots were analyzed using antibodies against Oct4 (sc-8628; Santa Cruz Biotechnology, Inc.), Nanog (ab80892; Abcam) or to GAPDH (mouse monoclonal; Sigma-Aldrich).

Protein transduction and transposon removal from primary iPSCs. The expression vector PCDH-cPP-mPB-HIS contains a nuclear localization sequence and protein transduction domain consisting of nine arginine residues (9R) cPP at the N-terminus and a HIS tag at the C-terminus of the transposase (mPB) protein. This expression cassette was assembled by standard PCR methods (13). Expression in 293T and purification of PCDH-cPP-mPB-HIS was performed as described previously (14). Protein transduction was performed with rat iPSCs on L-wnt3a feeder cells using $2 \mathrm{mM}$ recombinant protein for $6 \mathrm{~h}$. Approximately $6 \times 10^{5}$ cells were then seeded into $10-\mathrm{cm}$ dishes containing feeder cells following three days of culture. The next day, $0.2 \mu \mathrm{M}$ fialuridine (FIAU) was added and the selection was continued for 10 days. The resulting colonies were selected and expanded, and then the transposon was removed. Transposon removal was identified by genomic PCR. Primers for PCR are listed in Table II.

Differentiation of embryoid bodys (EBs). Aliquots of $25 \mu 1$ differentiation medium (10\% FBS; Invitrogen Life Technologies) containing 1,000 cells were cultivated in 'hanging drops' for 2 days and subsequently in suspension in petri dishes for an additional three days. The combination of bone morphogenetic protein-2 (BMP-2; $15 \mathrm{ng} / \mathrm{ml}$; Invitrogen Life Technologies) and tumor growth factor-1 (TGF-1; $2 \mathrm{ng} / \mathrm{ml}$; Invitrogen Life Technologies) which enhanced chondrogenic differentiation efficiency was added to the medium during the suspension phase. The EBs were plated onto $0.1 \%$ gelatin-coated 24-well plates for Alcian blue staining or immunostaining, and onto 35-mm dishes for total RNA isolation. Alcian blue staining was performed as described previously (15). Immunostaining and qPCR were performed as aforementioned. The primers for PCR are listed in Table III.

\section{Results}

Formation of iPSCs. Following 14 days, colonies with an embryonic stem (ES)-like morphology were observed in REFs transfected with PB-OSKM (Fig. 1A), which also stained positive with alkaline phosphatase (AP) (Fig. 1E). Following being supplemented with $1 \mu \mathrm{M}$ PD0325901, the colonies remained intact at 21 days (Fig. 1B). At 35 days, its shape was large and round with clear boundaries (Fig. 1C), which was very similar to the shape of rat ES (Fig. 1D). However, the empty vehicle control group (Fig. 1F) exhibited no clone formation at any time.

iPSC clone identification. In the present study, three clones were selected for examining the associated genes. The immunostaining (Fig. 2A) and western blotting results (Fig. 2B) revealed that the cells were positive for surface marker genes, including Oct4, Nanog and SSEA1. These findings indicated that the iPSCs were similar to rat ES regarding their biological characteristics. qPCR analysis (Fig. 2C) indicated that the clones exhibited lower expression levels of IRX3, Maf and Hoax 5 compared with REF, while Oct4, nanog, Ramp2, Sox 2 and Sal3 were expressed at higher levels in the clones 
A
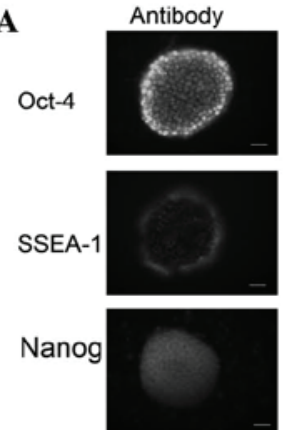

C
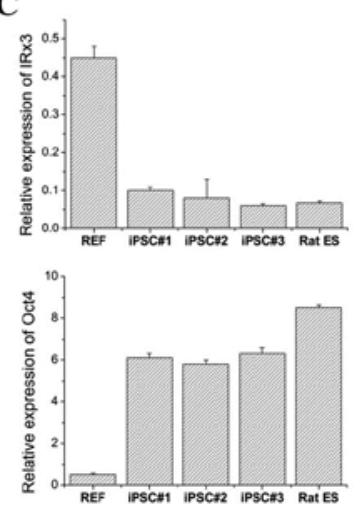

DAPI
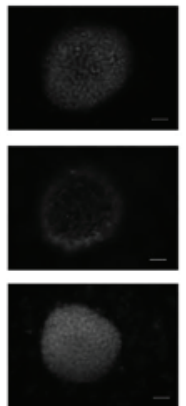

Merge
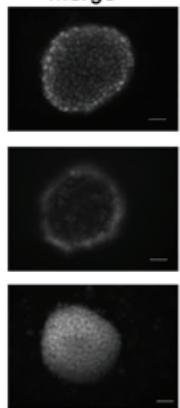
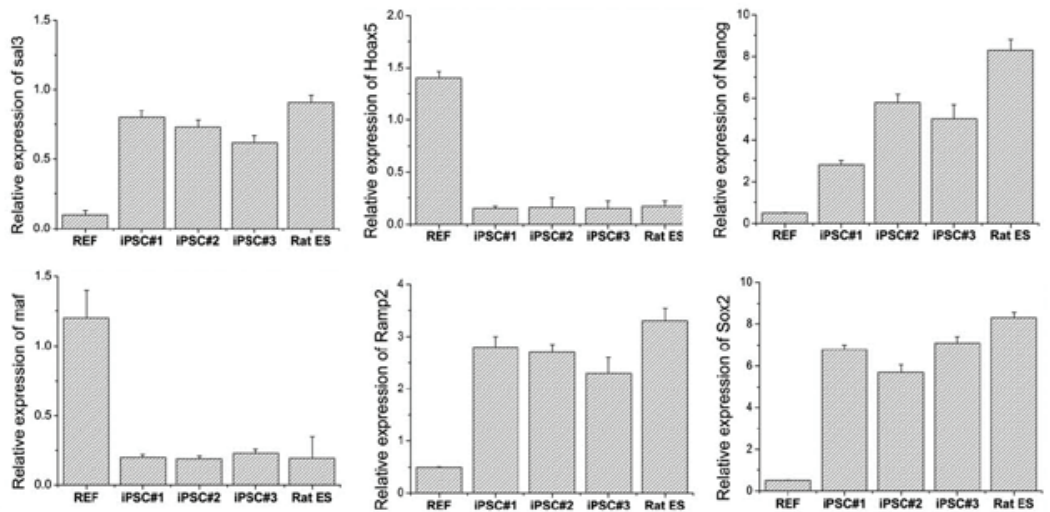

$\mathbf{B}$

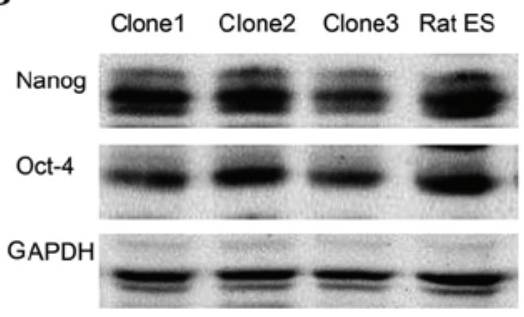

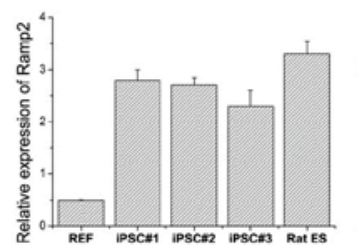

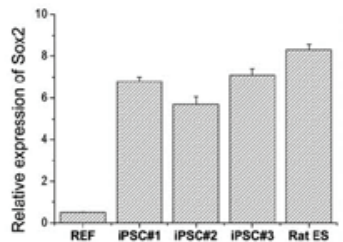

Figure 2. Induced pluripotent stem cells clone identification. (A) Immunostaining of Oct4, SSEA-1 and Nanog (bar, $20 \mu \mathrm{m}$ ); (B) Western blot analysis results; (C) reverse-transcription polymerase chain reaction results. ES, embryonic stem cells; REF, rat embryonic fibroblasts; IPSC, induced pluripotent stem cells; OCT-4, octamer-binding transcription factor 4; SSEA-1, stage-specific embryonic antigen 1.

compared with those of REF. These findings indicated that iPSCs expressed pluripotent markers and exhibited a similar gene expression to that of rat ES.

Transposon removal from primary iPSCs. In order to further examine whether the tranposase $\mathrm{mPB}$ system may enter into the cells to remove piggyBac-carried genes, the piggyBac-green fluorescent protein (GFP) plasmid was transduced into $293 \mathrm{~T}$ cells (Fig. 3A). Following $48 \mathrm{~h}, 0.2 \mu \mathrm{g} / \mathrm{ml}$ of the mPB protein was added for $8 \mathrm{~h}$. Approximately $70 \%$ of the GFP was removed following $48 \mathrm{~h}$ (Fig. 3B), indicating the $\mathrm{mPB}$ protein had the capability to remove piggyBac transposon-carrying genes efficiently. Following adding FIAU, the cells without gene removal gradually died, while the cells with gene removal were able to survive. Finally, the iPSC clones without genetic modification were obtained (Fig. 3C).

Chondrogenic differentiation of reprogrammed iPSCs. In EB outgrowths derived from iPSCs, numerous Alcian blue-stained regions were found, indicating the presence of acidic proteoglycans (Fig. 4A and B). Acidic proteoglycans were suggestive for cartilaginous tissue. The cells in these areas produced collagen II as demonstrated by immunostaining (Fig. 4C and D). qPCR analysis demonstrated that genes encoding transcription factors involved in mesenchymal differentiation, including extracellular matrix proteins of cartilage tissue (Type II collagen, aggrecan) were found to be expressed in EBs during culture (Fig. 4E). On the 20th day of culture time, the expression levels of Type II were highest.
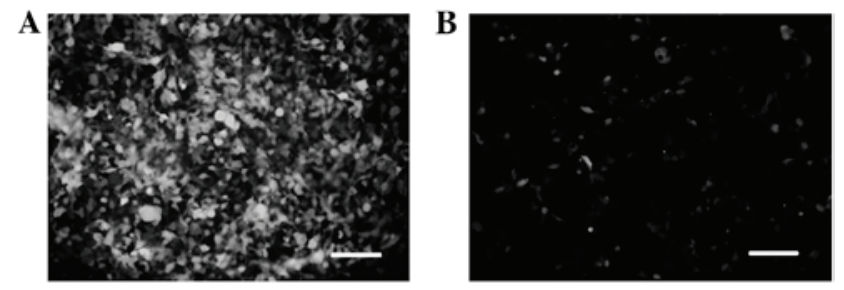

C

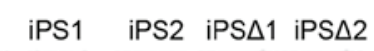

$\mathrm{L}$

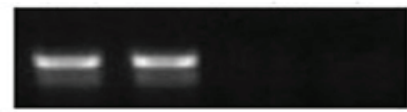

$\mathrm{R}$

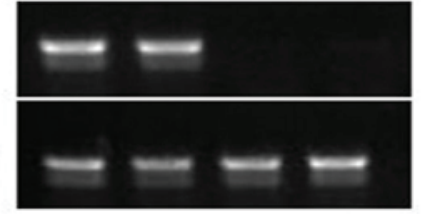

Control

Figure 3. Transposon removal from primary iPSCs. (A) PiggyBac-green fluorescent protein-controlled plasmid-tranfected 293T cells (bar, $100 \mu \mathrm{m}$ ); (B) $\mathrm{mPB}$ protein added to $293 \mathrm{~T}$ cells following $48 \mathrm{~h}$ (bar=100 $\mu \mathrm{m}$ ); (C) Genomic polymerase chain reaction analysis of RiPSC lines designed to detect the individual transposon integration sites. L (Sox2-K1f4 junction) and $\mathrm{R}$ (PGK promoter) denote the genome transposon junctions. RiPSC $\Delta$ indicates primary RiPSCs following removal of the transposon. iPSCs, induced pluripotent stem cells; RiPSC, Rat induced pluripotent stem cells.

\section{Discussion}

In the present study, piggyBac plasmid (carrying four or five 
genes) and transposase mPB system were applied to obtain rat iPSCs. Particularly, the transposase mPB system was used to successfully remove the transposons-carried genes to obtain the iPSCs without genetic modification. The preliminary study also revealed that iPSCs differentiate into chondrocytes in vitro.

Previously, the formation of iPSCs mostly depended on the mediation of virus (retrovirus, lentivirus and adenovirus). In 2006, Takahashi and Yamanaka demonstrated that retrovirus-mediated introduction of four transcription factors (Oct-3/4, Sox2, c-Myc and KLF4) into mouse embryonic or adult fibroblasts and selection for the expression of Fbx15, a target of Oct-3/4 and Sox2, resulted in the generation of iPSCs, which exhibited the morphology and growth properties of ES cells and expressed ES cell marker genes $(16,17)$. In 2009, Sommer et al (18) described the application of a single lentiviral vector expressing a 'stem cell cassette' composed of the four transcription factors and a combination of $2 \mathrm{~A}$ peptide and internal ribosome entry site technology, generating iPSCs from postnatal fibroblasts. Using a doxycycline-inducible lentiviral system, Maherali et al (19) developed a strategy to induce human iPSCs formation at high efficiency, and they obtained 'secondary' human iPSCs at a frequency of at least 100 -fold greater than the initial conversion upon addition of doxycycline to human iPSCs-derived differentiated cells. However, a major limitation of this technology by viral expression of the transcription factors is the use of potentially harmful genome-integrating viruses. Stadtfeld et al (20) obtained mouse iPSCs from fibroblasts and liver cells by using non-integrating adenoviruses transiently expressing Oct4, Sox 2, Klf4 and c-Myc. These adenoviral iPSCs revealed DNA demethylation characteristic of reprogrammed cells, expressed endogenous pluripotency genes, formed teratomas and contributed to multiple tissues. However, viral integration into the host genome increases the risk of tumorigenicity, hindering its clinical applications (21). In order to avoid the use of a viral vectors, Okita et al $(12,22)$ described an alternative method to generate iPSCs from MEF by continual transfection of plasmid vectors. However, the reprogramming efficiency of this protocol was very low. Previously, Yusa et al (13) reported an efficient piggyBac transposon-based approach to generate integration-free iPSCs in MEF. In the present study, piggyBac transposon-based reprogramming was successfully used to generate iPSCs in REF. Furthermore, PCDH-cPP-mPB-HIS was applied to successfully remove the piggyBac transposon, to obtain iPSCs clones without any genetic alteration.

Pluripotent mouse ES cells have been derived and maintained by using various empirical combinations of feeder cells, conditioned media, cytokines, growth factors, hormones, fetal calf serum and serum extracts. It has been reported that ES cells have an innate program for self-replication, which does not require extrinsic instruction (23). The self-renewal factors present in embryonic carcinoma cell-derived conditioned medium may be responsible for the self-renewal capacity of embryonic carcinoma and ES cells independently of leukemia inhibitory factor signaling (24). In the present study, the L-wnt3a feeding layer was applied to enhance ES cell adherence. In the culture of iPSCs, L-wnt3a with PD0325901 was able to maintain its pluripotent state. These findings indicated that Wnt3a activated Wnt in the conventional way to maintain its self-renewal.
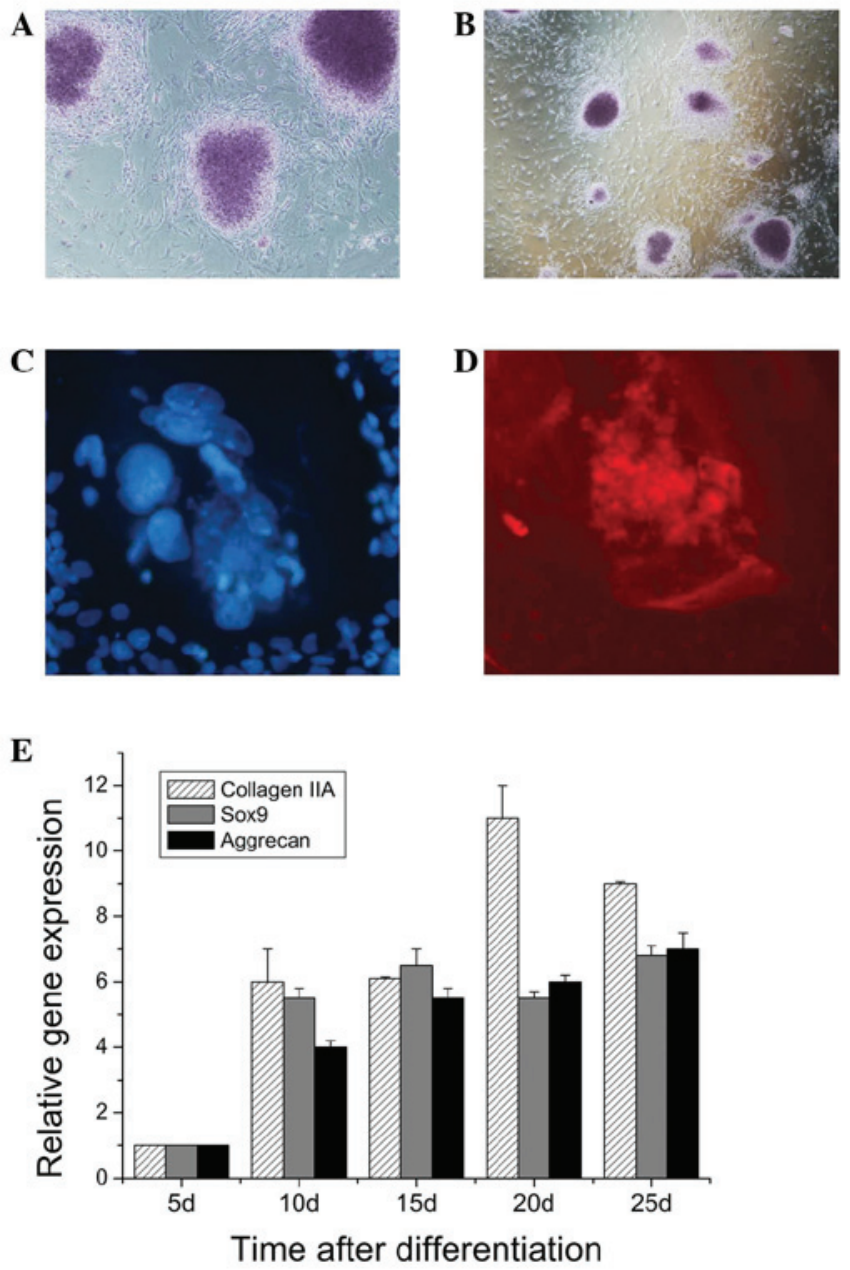

Figure 4. Chondrogenic differentiation of reprogrammed iPSCs. (A) Alcian blue-stained areas in a three-day EB outgrowth (magnification, x400); (B) Alcian blue-stained areas in a three-day EB outgrowth (magnification, $\mathrm{x} 100$ ); (C) Immunostaining for collagen type II: nuclei were counterstained with DAPI (magnification, x400); (D) Immunostaining for collagen type II. These cells were embedded in a matrix which stained positive with antibodies against collagen II (bar, $50 \mu \mathrm{m}$; magnification, $\mathrm{x} 400$ ); (E) expression of collagen IIA, Sox 9 and aggrecan during differentiation of iPSCs. iPSCs, induced pluripotent stem cells; EB, embryoid body.

Generally, chondrogenic differentiation of iPSCs may be achieved in EBs by co-culturing with chondrocytes or adding selected growth factors to the medium. Qu et al (25) studied whether co-culture with primary chondrocytes induces human ES cells or iPSCs to differentiate into chondrocyte lineages, and their results demonstrated that co-culture of human ES cells or iPSCs with primary chondrocytes induced specific chondrogenic differentiation. Wei et al (26) used a lentivirus to transduce iPSCs seeded in an alginate matrix with TGF- $\beta 1$ and then co-cultured these iPSCs with chondrocytes in vitro. The authors observed an increased expression of cartilage-associated genes, including collagen II, aggrecan and cartilage oligomeric matrix protein, and decreased gene expression of the degenerative cartilage marker, vascular endothelial growth factor and the histological results also revealed a dense sulphated extracellular matrix in the co-culture of TGF- $\beta 1$-transfected iPSCs with chondrocytes in the alginate matrix. Kuboth et al (27) demonstrated the induction of chondrogenic iPSCs differentiation by certain members of 
the TGF- $\beta$ family (BMP-2, TGF- $\beta 1$ ). Their results demonstrated that the number of Alcian blue-positive nodules and the expression of cartilage marker molecule collagen type II increased following application of BMP-2. In the present study, the combination of BMP- 2 and TGF- $\beta 1$ was used to enhance chondrogenic differentiation of iPSCs. The results revealed that extracellular matrix proteins of cartilage tissue (Type II collagen) were found to be highly expressed in EBs during culture. The gene expression analysis demonstrated that the established iPSCs differentiated into chondrocytes with increased expression of cartilage-associated genes, including collagen II and aggrecan.

Koyama et al (28) examined the chondrogenic differentiation capability of human iPSCs using a multistep culture method consisting of EB formation, cell outgrowth from EBs, monolayer culture of sprouted cells from EBs and 3-dimensional pellet culture. The authors identified that the cells in pellets exhibited a spherical morphology typical of chondrocytes and were surrounded by extracellular matrix that contained acidic proteoglycans following 2-3 weeks of pellet culture, and the expression of type II collagen and aggrecan in pellets progressively increased. Furthermore, BMP-2 treatment of iPSC-derived MSC-like micromass cultures resulted in a phenotype more typical of articular chondrocytes compared with the pellet culture differentiation, characterized by the enrichment of cartilage-specific type II collagen, decreased expression of type I collagen as well as lack of chondrocyte hypertrophy (29). In the present study, cells were cultivated in 'hanging drops' for two days and subsequently in suspension in petri dishes for an additional three days. In the EB outgrowths derived from iPSCs, numerous Alcian blue-stained regions were found, indicating the presence of cartilaginous tissue.

In conclusion, the present study indicated that iPSCs may be generated by applying the piggyBac plasmid (carrying four or five genes) and the transposase mPB system. Particularly, the transposase mPB system was used to successfully remove the transposons-carried genes to obtain rat iPSCs without genetic modification. Additionally, the present study also revealed that the iPSCs were able to differentiate into chondrocytes in vitro.

\section{References}

1. Roelofs AJ, Rocke JP and De Bari C: Cell-based approaches to joint surface repair: a research perspective. Osteoarthritis Cartilage 21: 892-900, 2013.

2. Teramura T, Onodera Y,Mihara T, et al: Induction of mesenchymal progenitor cells with chondrogenic property from mouse-induced pluripotent stem cells. Cell Reprogram 12: 249-261, 2010.

3. Takahashi K, Tanabe K, Ohnuki M, et al: Induction of pluripotent stem cells from adult human fibroblasts by defined factors. Cell 131: 861-872, 2007.

4. Szabo E, Rampalli S, Risueno RM, et al: Direct conversion of human fibroblasts to multilineage blood progenitors. Nature 468: 521-526, 2010.

5. Efe JA, Hilcove S, Kim J, et al: Conversion of mouse fibroblasts into cardiomyocytes using a direct reprogramming strategy. Nat Cell Biol 13: 215-222, 2011.

6. Kim J, Efe JA, Zhu S, et al: Direct reprogramming of mouse fibroblasts to neural progenitors. Proc Natl Acad Sci USA 108 7838-7843, 2011.
7. Jang YY, Collector MI, Baylin SB, Diehl AM and Sharkis SJ: Hematopoietic stem cells convert into liver cells within days without fusion. Nat Cell Biol 6: 532-539, 2004.

8. Yu B, He ZY, You P, et al: Reprogramming fibroblasts into bipotential hepatic stem cells by defined factors. Cell Stem Cell 13: 328-340, 2013.

9. Hanna J, Wernig M, Markoulaki S, et al: Treatment of sickle cell anemia mouse model with iPS cells generated from autologous skin. Science 318: 1920-1923, 2007.

10. Wernig M, Zhao JP, Pruszak J, et al: Neurons derived from reprogrammed fibroblasts functionally integrate into the fetal brain and improve symptoms of rats with Parkinson's disease. Proc Natl Acad Sci USA 105: 5856-5861, 2008.

11. Nishimura K, Nakagawa T, Ono K, et al: Transplantation of mouse induced pluripotent stem cells into the cochlea. Neuroreport 20: 1250-1254, 2009.

12. Okita K, Nakagawa M, Hyenjong H, Ichisaka T and Yamanaka S: Generation of mouse induced pluripotent stem cells without viral vectors. Science 322: 949-953, 2008

13. Yusa K, Rad R, Takeda J and Bradley A: Generation of transgene-free induced pluripotent mouse stem cells by the piggyBac transposon. Nat Methods 6: 363-369, 2009.

14. Peitz M, Pfannkuche K, Rajewsky K and Edenhofer F: Ability of the hydrophobic FGF and basic TAT peptides to promote cellular uptake of recombinant Cre recombinase: a tool for efficient genetic engineering of mammalian genomes. Proc Natl Acad Sci USA 99: 4489-4494, 2002.

15. Kramer J, Hegert C, Guan K, et al: Embryonic stem cell-derived chondrogenic differentiation in vitro: activation by BMP-2 and BMP-4. Mech Dev 92: 193-205, 2000.

16. Takahashi K and Yamanaka S: Induction of pluripotent stem cells from mouse embryonic and adult fibroblast cultures by defined factors. Cell 126: 663-676, 2006.

17. Yamanaka S: Strategies and new developments in the generation of patient-specific pluripotent stem cells. Cell Stem Cell 1: 39-49, 2007.

18. Sommer CA, Stadtfeld M, Murphy GJ, et al: Induced pluripotent stem cell generation using a single lentiviral stem cell cassette. Stem Cells 27: 543-549, 2009.

19. Maherali N, Ahfeldt T, Rigamonti A, et al: A high-efficiency system for the generation and study of human induced pluripotent stem cells. Cell Stem Cell 3: 340-345, 2008.

20. Stadtfeld M, Nagaya M, Utikal J, Weir G and Hochedlinger K: Induced pluripotent stem cells generated without viral integration. Science 322: 945-949, 2008

21. Nakagawa M, Koyanagi M, Tanabe $\mathrm{K}$, et al: Generation of induced pluripotent stem cells without Myc from mouse and human fibroblasts. Nat Biotechnol 26: 101-106, 2008.

22. Okita K, Hong H, Takahashi K and Yamanaka S: Generation of mouse-induced pluripotent stem cells with plasmid vectors. Nat Protoc 5: 418-428, 2010.

23. Ying QL, Wray J, Nichols J, et al: The ground state of embryonic stem cell self-renewal. Nature 453: 519-523, 2008.

24. Kawazoe S, Ikeda N, Miki K, et al: Extrinsic factors derived from mouse embryonal carcinoma cell lines maintain pluripotency of mouse embryonic stem cells through a novel signal pathway. Dev Growth Differ 51: 81-93, 2009.

25. Qu C, Puttonen KA, Lindeberg H, et al: Chondrogenic differentiation of human pluripotent stem cells in chondrocyte co-culture. Int J Biochem Cell Biol 45: 1802-1812, 2013.

26. Wei Y, Zeng W, Wan R, et al: Chondrogenic differentiation of induced pluripotent stem cells from osteoarthritic chondrocytes in alginate matrix. Eur Cell Mater 23: 1-12, 2012.

27. Kuboth S, Kramer J and Rohwedel J: Chondrogenic differentiation in vitro of murine two-factor induced pluripotent stem cells is comparable to murine embryonic stem cells. Cells Tissues Organs 196: 481-489, 2012.

28. Koyama N, Miura M, Nakao K, et al: Human induced pluripotent stem cells differentiated into chondrogenic lineage via generation of mesenchymal progenitor cells. Stem Cells Dev 22: 102-113, 2013.

29. Guzzo RM, Gibson J, Xu RH, Lee FY and Drissi H: Efficient differentiation of human iPSC-derived mesenchymal stem cells to chondroprogenitor cells. J Cell Biochem 114: 480-490, 2013. 